\title{
Prospective clinical study of the skin markers in childhood obesity-an emerging pandemic
}

\author{
Dash Mrutunjay ${ }^{1}$, Jena A. $\mathrm{K}^{2}$, Panda $\mathrm{M}^{3}$, Patro $\mathrm{N}^{4}$ \\ ${ }^{1}$ Dr. Mrutunjay Dash, Associate Professor, Department of Pediatrics, ${ }^{2}$ Dr. Ajaya Kumar Jena, Associate Professor, \\ Department of Skin and VD, ${ }^{3}$ Dr. Maitreyee Panda, Assistant Professor, Department of Dermatology, AIIMS \\ Bhubaneswar, ${ }^{4}$ Dr. Nibedita Patro, Assistant Professor, Department of Skin and VD, IMS and Sum Hospital, \\ Bhubaneswar. Odishsa, India
}

Address for correspondence: Dr. Mrutunjay Dash, Email: mrutyunjaydash@soauniversity.ac.in

\begin{abstract}
Introduction: Obesity poses an alarming threat to the children in recent days due to poor lifestyle and improper dietary habits. These children are at higher risk of developing Coronary artery diseases, Hypertension, Diabetes mellitus in their early thirties and emerge as an important public health problem. Childhood obesity manifests with a wide array of dermatological changes. In this study we analyzed the dermatological manifestations in the obese and overweight children in the age range of 5-14 years. Methods: All the children taken into the study were either obese or overweight as per WHO criteria of BMI according to the height and weight. Triceps skin fold thickness and waist circumference of these children were also taken for confirmation of obesity. Skin manifestations in these children were diagnosed by the dermatologists and plotted in graph. Results: Total 76 children were taken into the study out of which Acanthosis nigricans is the most common manifestation in the age group of 10-14 years and infections are most common in the age group of 5-9 years. Conclusion: The skin manifestations are the markers of chronic obesity in childhood having negative impact on the quality of life and imparting low self esteem among the peer group.
\end{abstract}

Key words: Obesity, Acanthosis Nigricans, Striae Distensae, Intertrigo

\section{Introduction}

Obesity and overweight issues in childhood is nothing less than an emerging pandemic globally and the trend is increasing phenomenally in developing countries. In India a sizeable fraction of children are recorded being overweight and obese, and the reason behind this current scenario is attributable to the changing lifestyle preferences and cultural environment on a background of predominant familial predisposition. There is a significant association of physical and psychological impairment with this condition, such as social rejection and teasing by their peer groups ending in low self esteem [1,2].

The WHO center for disease control and prevention defined obesity as above $95 \%$ percentile of body mass Index (BMI) for age and sex and overweight as between $85^{\text {th }}$ to $95^{\text {th }}$ percentile of BMI for age and sex. $[3,4,5]$.

Manuscript received: $24^{\text {th }}$ May 2016

Reviewed: $6^{\text {th }}$ June 2016

Author Corrected; $16^{\text {th }}$ June 2016

Accepted for Publication: $29^{\text {th }}$ June 2016
As the BMI does not distinguish between fat and muscle mass it is not a very sensitive measure of the percentage of body fat. More accurate methods to measure the percentage of body fat includes underwater weighing (densitometry), Multi-frequency bioelectrical impedance analysis (BIA), and magnetic resonance imaging (MRI) [6]. Clinically assessment of obesity can be done by waist circumference and skin fold thickness apart from BMI. The cutaneous changes are highly prevalent in obese individuals and the skin manifestation includes acne, acanthosis nigricans, striaedistensae, sebborhoea, keratosis pilaris, plantar hyperkeratosis, stasis dermatitis etc $[7,8]$.

There is also increased incidence of skin infections like folliculitis, erysipelas, impetigo, fungal infections and mixed infections like Intertrigo. Conditions like Psoriasis, atopic dermatitis and hidradenitis suppurativa have a complex interaction with obesity and exhibit a worsened course. 
Excessive fat deposition is interlinked to hyperandrogenism, abnormal glucose intolerance, insulin resistance, inflammation, hyperlipidemia, hypertension, infertility, cardiovascular complications etc. mainly manifesting in adults $[9,10]$.

Our study is a prospective cross sectional study to determine the prevalence of skin manifestations in overweight and obese patients of the pediatric age group.

\section{Material and Methods}

A prospective cross sectional study was conducted in the department of pediatrics in collaboration with the department of dermatology in a tertiary care teaching hospital.

Duration of study was 1 year from January 2015 to December 2015. Patients enrolled in the study were otherwise healthy non diabetic children between 5-14 Years fulfilling the $\mathrm{CDC}$ criteria of obesity and overweight.

Exclusion criteria: All the children with BMI less than 85 percentile for the appropriate age and sex were excluded from the study.

Children with BMI more than 85 percentile for the appropriate age and sex having abnormal endocrinological or congenital abnormalities were also excluded from the study.

Detailed medical and family history and medication records were taken. The clinical evaluation included Anthropometric measurements of weight and height, BMI, Waist circumference and skin fold thickness. Patients who were diagnosed as overweight and obese were subjected to dermatological examination. Children with skin manifestation were enrolled in our study and referred to the dermatology OPD for detailed examination, evaluation and management.

The detailed dermatological examination was done involving the skin, mucosa, muco-cutaneous junction, scalp and hair, nails and palms and soles. All the data were recorded and statistically analyzed.

Anthropometric Measurement

1. Height measurement

- Height of the child was measured using a measuring scale attached to the wall.

- The child was made to stand with heels together in standing position, with shoes removed and buttocks, shoulder \& occiput touching the wall.

- Accurate reading to the nearest centimeter was taken

2. Weight Measurement

- Weight of the child was taken in a digital weighing machine with range of $100 \mathrm{gms}$ in a standing condition with light clothes and shoes removed.

\section{Waist Circumference}

- The midpoint of the lower rib and the upper most part of the iliac crest was marked on both sides of the trunk, both these points were joined by a measuring tape and the waist circumference was recorded.

4. Tricep skin fold thickness was recorded using a caliper on the non-dominant upper arm.

\section{Results}

76 children within the age range of 5-14 years were taken into the study those fulfilling the criteria of CDC of either overweight or obese. (According to BMI).

There were $40(52 \%)$ boys and $36(47.47 \%)$ girls. 22 children were obese according to their BMI as per their age and sex and rest 54 were overweight. Children in the age group of 5-9 years were 53 and those in the age group of 10-14 were 23 . (Table1).

Table-1: Age and sex distribution of the children.

\begin{tabular}{|c|c|c|c|c|c|c|c|c|c|c|}
\hline \multirow{2}{*}{ Sl. No } & \multicolumn{2}{|c|}{ No. of Patients } & \multicolumn{2}{|c|}{$5-10$} & \multicolumn{2}{c|}{$11-14$} & \multicolumn{2}{c|}{$\%$ of (5-10) Years } & \multicolumn{2}{c|}{ of (11-14) Years } \\
\cline { 2 - 11 } & Boys & Girls & Boys & Girls & Boys & Girls & Boys & Girls & Boys & Girls \\
\hline 1. & 40 & 36 & 29 & 24 & 11 & 12 & 72.5 & 66.6 & 27.5 & 33.3 \\
\hline
\end{tabular}


Table-2: Prevalence of Skin manifestations in Children.

\begin{tabular}{|c|c|}
\hline Disease & Number of cases \\
\hline Acanthosis Nigricans (AN) & 26 \\
\hline Infections(I) & 21 \\
\hline Striae Distensae(SD) & 15 \\
\hline Acne Vulgaris(AV) & 7 \\
\hline Intertrigo(INT) & 10 \\
\hline Plantar Hyperkeratosis(PHK) & 5 \\
\hline Keratosis Pilaris(KP) & 4 \\
\hline Atopic dermatitis(AD) & 3 \\
\hline Seborrhoea(S) & 7 \\
\hline Alopecia(A) & 1 \\
\hline Hirsutism(H) & 2 \\
\hline
\end{tabular}

Skin manifestations were examined clinically by the dermatologists and their prevalence was documented. (Table2). It was found that the most common manifestation was acanthosis nigricans (AN) followed by superficial infections. Among all skin manifestations AN (Fig-1)was found in around 30\% cases followed by infections like Folliculitis, Furunculosis (Fig-2), Tinea Corporis. Around 20\% pts had Striae distensae and 10\% had Acne vulgaris (Fig 3, 4). We also found 2 cases of hirsutism in the age group of 10-14 years. Overall within the age group of 5-9 years Superficial skin infections were the commonest and those in the age group of 10-14 years AN was the commonest dermatological manifestations. (Table-3).

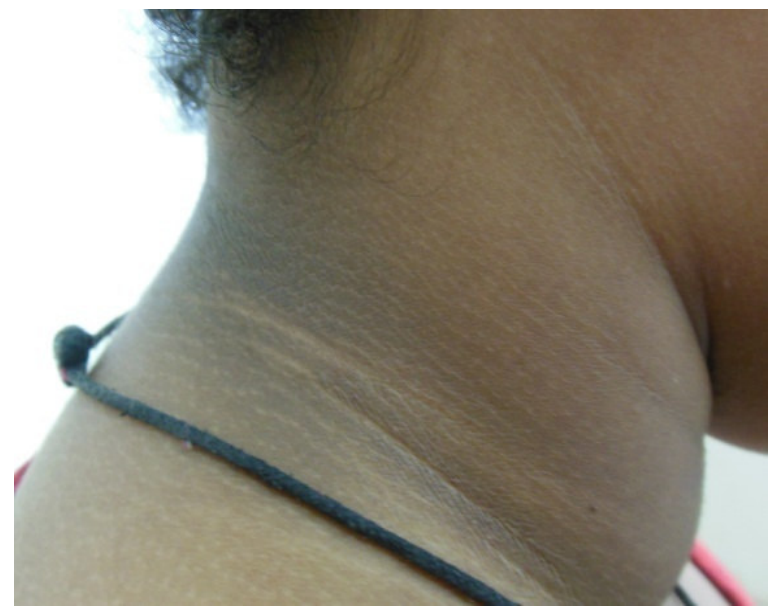

Fig.-1: Acanthosis Nigricans

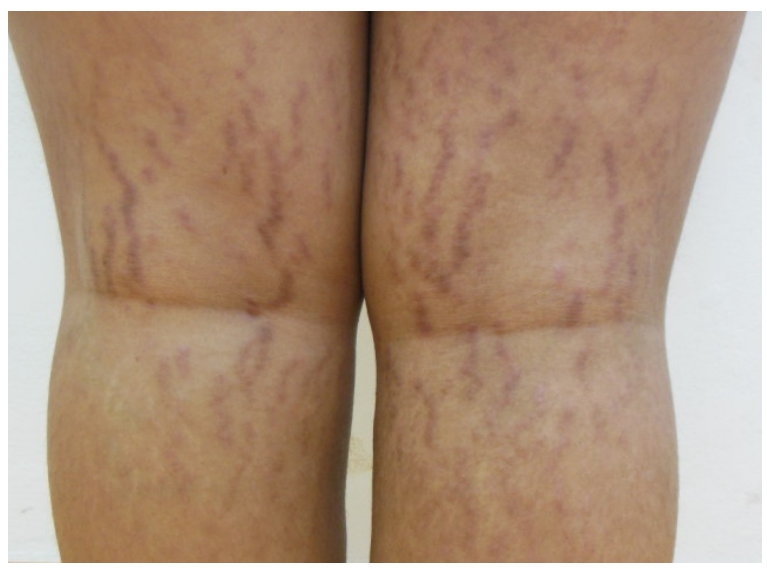

Fig. 3: Striae Distensae

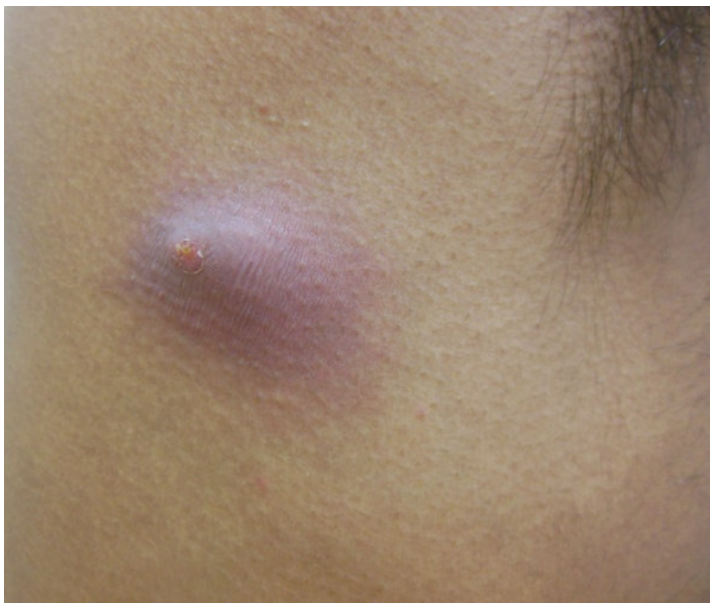

Fig.-2: Furunculosis

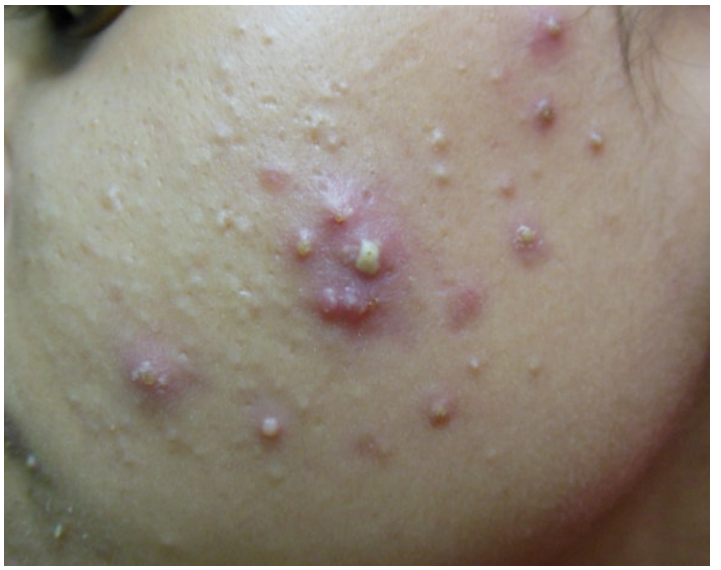

Fig.- 4: Acne Vulgaris 
Table-3: Prevalence of skin manifestation in obese and overweight children.

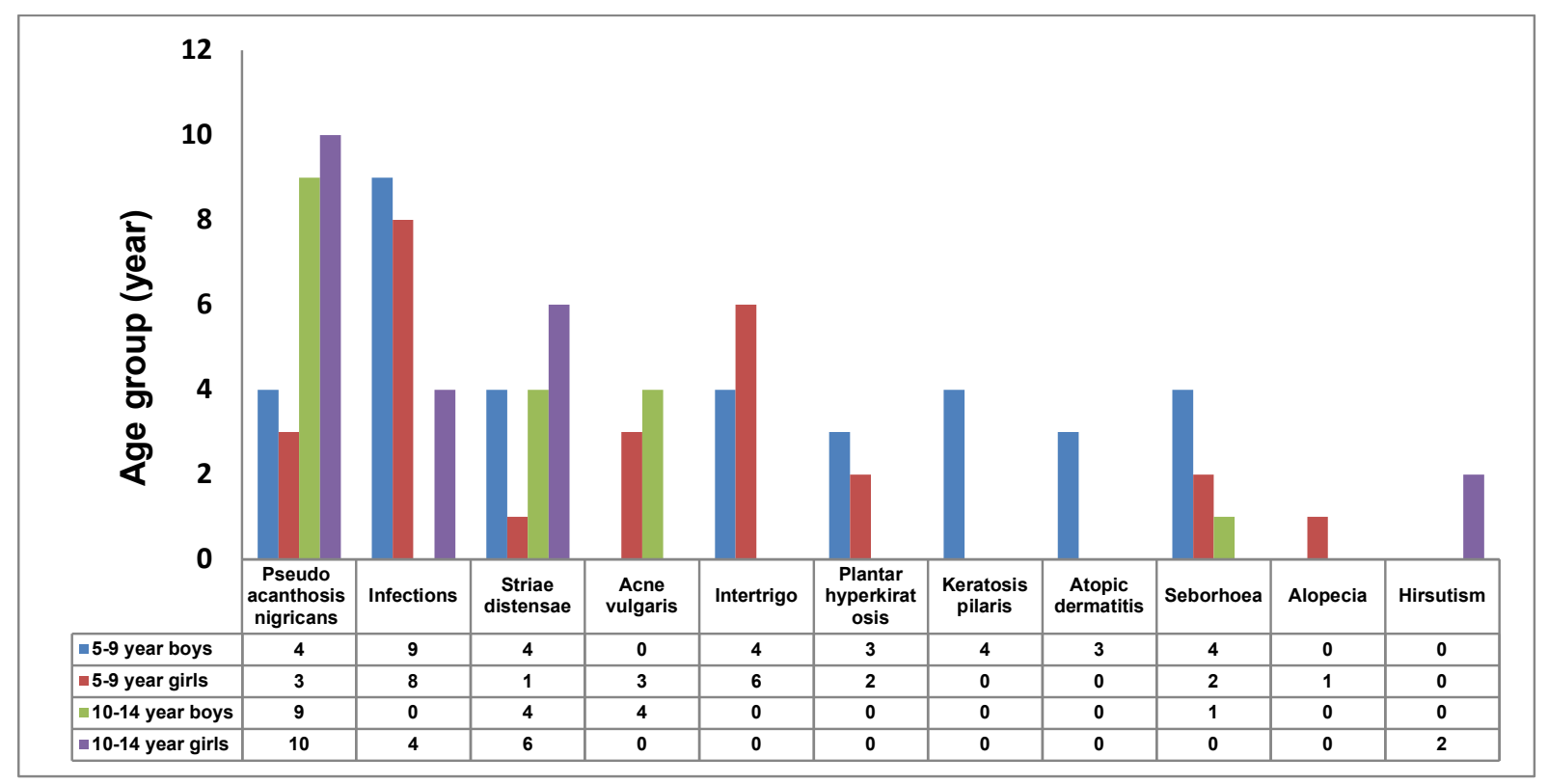

\section{Discussion}

Childhood obesity is an important predictor of increased morbidity and health issues in adulthood. The rising incidence of this health problem is slowly gripping the developing countries and becoming an important public health issue putting immense adverse physical, psychological and social impact in the society.

Obesity is commonly associated with many dermatological manifestations. In our study we encountered a wide spectrum of skin diseases in obese and overweight children. The effect of obesity on skin includes alteration in the barrier functions, increased sebaceous and sweat gland activities, decreased lymphatic flow, increased cutaneous vascular supply, alteration in collagen structures and wound healing $[7,8]$. These changes are responsible for increased risk of skin infections, lymphedema and venous insufficiency.

In our study, the incidences of bacterial and fungal infections are more in relation to excessive skin folds leading to occlusion and maceration. Folliculitis, a superficial bacterial infection of the hair follicle commonly infected by staphylococcus aureus has the highest prevalence among the infections in our study specially in the age group of 5-9 yrs, irrespective of the gender. Intertrigo and Dermatophytic fungal infection also accounts to a significant proportion among all the infections. These findings are comparable to the study conducted by Al-Saeed WY et al [9].
Insulin Resistance and androgen excess has a significant risk of developing Acne, Acanthosis nigricans, Hirsutism, Sebborhoea, Alopecia etc. The stretching of the skin leads to striaedistensae and excessive body weight contributes to plantar Hyperkeratosis. [8, 9, 10]. Acanthosis nigricans (AN) was the most prevalent manifestation among all reported children, mostly with $10-14$ year girls. AN was the most common disease with the average BMI value 24.4 for 10- 14 year boys as well as, BMI value of 25.1 of 10-14 year girls. Striae distensae (SD) was most prevalent among 10-14 year girls.

In a comparative study conducted by Boza et al [8] they found statistically significant relationship between the skin manifestations and obesity as compared with the control groups. The presence of striae, AN and bacterial infections were also found to have a co-relation with degree of obesity. In their study skin manifestations of acrochordons and lymphedema were found which is not documented in our study.

Divayshree et al [7] studied the various cutaneous manifestations in obese adults and analyzed the relationship with BMI in 100 cases. They found majority of patients (68\%) had BMI of 30.000-34.99, while $23 \%$ had BMI of 35.00- 39.99, and 9\% had BMI of $>=40.00$. The skin conditions associated with obesity were skin tags, AN, plantar hyperkeratosis, striae and infections. Overall they found fungal 
infections and intertrigo to be more common among all infections, but in our study we found bacterial infections predominantly. Only few studies have been published till date showing the association of skin manifestations with childhood obesity.

\section{Conclusion}

This study reflects the high incidence of skin diseases in obese and overweight children. Measures should be taken for adequate health education and counseling. The parents should be aware of the importance of healthy lifestyle and right nutritional choices to reduce the burden of childhood obesity in the society. Early identification and management of these skin conditions can significantly improve the quality of life among these children.

\section{Funding: Nil, Conflict of interest: Nil \\ Permission from IRB: Yes}

\section{References}

1. Flegal KM, Wei R, Ogden CL, Freedman DS, Johnson CL, Curtin LR. Characterizing extreme values of body mass index-for-age by using the 2000 Centers for Disease Control and Prevention growth charts. Am J Clin Nutr. 2009 Nov; 90(5):1314-20.doi: 10. 3945 /ajcn.2009.28335. Epub 2009 Sep 23.

2. Himes JH, Dietz WH. Guidelines for overweight in adolescent preventive services: recommendations from an expert committee. The Expert Committee on Clinical Guidelines for Overweight in Adolescent Preventive Services. Am J ClinNutr. 1994 Feb;59(2):307-16.

3. Dehghan M, Akhtar-Danesh N, Merchant AT. Childhood obesity, prevalence and prevention. Nutr J. 2005 Sep 2;4:24.
4. Burns T. Rook's Textbook of Dermatology. $8^{\text {th }}$ ed. Wiley, New Jersey. 2010.

5.Mirmirani P, Carpenter DM. Skin disorders associated with obesity in children and adolescents : a population-based study. Pediatr Dermatol. 2014 MarApr;31(2):183-90. doi: 10.1111/pde. 12271. Epub 2013 Dec 13.

6. G Hidalgo L. Dermatological complications of obesity. Am J ClinDermatol. 2002;3(7):497-506.

7. Divyashree R A, Naveen K N, Pai VV, Athanikar SB, Gupta G.Cutaneous manifestations of obesity among dermatology patients in a tertiary care center. Indian J Dermatol VenereolLeprol. 2014 May-Jun;80 (3):278. doi: 10.4103/0378-6323.132270.

8. Boza JC, Trindade EN, Peruzzo J, Sachett L, Rech L, Cestari TF. Skin manifestations of obesity: a comparative study. J Eur Acad Dermatol Venereol. 2012 Oct; 26 (10) : 1220-3. doi: 10. 1111/ j. 1468 - 3083. 2011. 04265 . x. Epub 2011 Sep 20.

9.Al-Saeed WY, Al-Dawood KM, Bukhari IA, Bahnassy A. Dermatoses in obese female schoolchildren in the Al-khobar area, eastern saudiarabia. J Family Community Med. 2006 May; 13(2):65-9.

10. Winkleby MA, Taylor CB, Jatulis D, Fortmann SP The long-term effects of a cardiovascular disease prevention trial: the Stanford Five-City Project Am J Public Health. 1996 Dec; 86(12):1773-9.

\section{How to cite this article?}

Dash Mrutunjay, Jena A. K, Panda M, Patro N. Prospective clinical study of the skin markers in childhood obesity-an emerging pandemic. Int J Pediatr Res.2016;3(6):462-466.doi:10.17511/ijpr.2016.i06.15. 